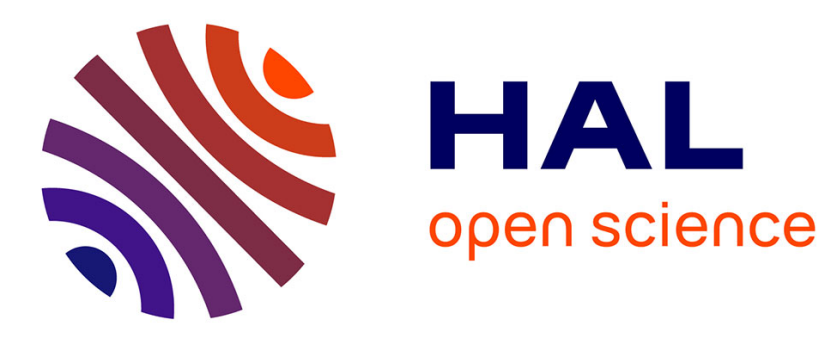

\title{
Stable Physical Human-Robot Interaction Using Fractional Order Admittance Control
}

\author{
Yusuf Aydin, Ozan Tokatli, Volkan Patoglu, Cagatay Basdogan
}

\section{To cite this version:}

Yusuf Aydin, Ozan Tokatli, Volkan Patoglu, Cagatay Basdogan. Stable Physical Human-Robot Interaction Using Fractional Order Admittance Control. IEEE Transactions on Haptics (ToH), 2018, 11 (3), pp.464-475. 10.1109/TOH.2018.2810871 . hal-03177201

\section{HAL Id: hal-03177201 \\ https://hal.science/hal-03177201}

Submitted on 23 Mar 2021

HAL is a multi-disciplinary open access archive for the deposit and dissemination of scientific research documents, whether they are published or not. The documents may come from teaching and research institutions in France or abroad, or from public or private research centers.
L'archive ouverte pluridisciplinaire HAL, est destinée au dépôt et à la diffusion de documents scientifiques de niveau recherche, publiés ou non, émanant des établissements d'enseignement et de recherche français ou étrangers, des laboratoires publics ou privés. 


\title{
Stable Physical Human-Robot Interaction Using Fractional Order Admittance Control
}

\author{
Yusuf Aydin, Student Member, IEEE, Ozan Tokatli, Member, IEEE, Volkan Patoglu, Member, IEEE, \\ and Cagatay Basdogan, Member, IEEE
}

\begin{abstract}
In the near future, humans and robots are expected to perform collaborative tasks involving physical interaction in various environments, such as homes, hospitals, and factories. Robots are good at precision, strength, and repetition, while humans are better at cognitive tasks. The concept, known as physical human-robot interaction (pHRI), takes advantage of these abilities and is highly beneficial by bringing speed, flexibility, and ergonomics to the execution of complex tasks. Current research in pHRI focuses on designing controllers and developing new methods which offer a better tradeoff between robust stability and high interaction performance. In this paper, we propose a new controller, fractional order admittance controller, for pHRI systems. The stability and transparency analyses of the new control system are performed computationally with human-in-the-loop. Impedance matching is proposed to map fractional order control parameters to integer order ones, and then the stability robustness of the system is studied analytically. Furthermore, the interaction performance is investigated experimentally through two human subject studies involving continuous contact with linear and nonlinear viscoelastic environments. The results indicate that the fractional order admittance controller can be made more robust and transparent than the integer order admittance controller and the use of fractional order term can reduce the human effort during tasks involving contact interactions with environment.
\end{abstract}

Index Terms-Physical human-robot interaction (pHRI), fractional order admittance control, robustness, stability, effective impedance, impedance matching, contact interactions, needle insertion.

\section{INTRODUCTION}

$\mathrm{R}$ EPEATABILITY and accuracy of robots are, typically, superior to humans; however, the problem solving skills and adaptability of humans are unmatched. Integrating the benefits of human and robot into tasks involving physical interaction, pHRI, may result in a significant increase in the overall performance. Such collaborative tasks require human and robot to be in the same environment and interact with each other throughout the operation. For instance, in a home or an office setting, a robot may collaborate with a human user to assemble or move furniture [1]. In a factory,

- Yusuf Aydin and Cagatay Basdogan are with College of Engineering, Koc University, Sariyer, Istanbul, 34450, Turkey. E-mail: yaydin@ku.edu.tr, cbasdogan@ku.edu.tr

- Ozan Tokatli is with Biomedical Engineering, University of Reading, Whiteknights, RG6 6AX, UK. Email: o.tokatli@reading.ac.uk

- Volkan Patoglu is with Faculty of Engineering and Natural Sciences, Sabanci University, Istanbul, 34956, Turkey. Email: vpatoglu@sabanciuniv.edu a robot and a worker may install a windshield on a car together [2]. In a hospital, a robot may work with a surgeon to operate on a patient. In such scenarios, in addition to being safe, the robot should maintain the stability of the coupled system under changes in both environmental conditions and human dynamical behaviour, while helping human to achieve a high task performance. Seeming trivial, the contradicting nature of stability and high performance requirements creates challenges for researchers.

To regulate a physical interaction between human and robot, impedance/admittance based controllers have been widely used [3]. An impedance controller computes forces to be applied by a robot for an imposed motion, while an admittance controller computes the trajectory to be followed by a robot under the action of forces applied. In general, it is more natural to utilize admittance control when robots used in pHRI tasks do not posses high backdrivability. Such robots are usually motion controlled; hence, the interaction force between human and robot serves as the input to an admittance controller that generates velocity references for the motion controller of the robot. In a typical implementation for pHRI, an admittance controller regulates the dynamics of interaction through a linear admittance model that consists of inertial and dissipative elements. Spring elements are not preferred for collaborative manipulation tasks as they force the robot to return to an equilibrium position, which is typically not desirable.

In this paper, we propose a fractional order admittance controller (FOAC) for pHRI. This control scheme relies on the fractional order calculus, which allows the use of integrators/differentiators of arbitrary orders. Hence, in addition to adjusting the usual admittance controller parameters (i.e. admittance mass and damping), one can also change the output response characteristics of the closed-loop system by changing the order of integration. In particular, order of integration enables more effective control of frequencydependent response of FOAC and brings more flexibility to adjust the trade-off between stability and transparency, making it well-suited for applications that involve pHRI. In this paper, the stability and robustness of such a controller and its effect on human effort and task performance during contact interactions with linear and nonlinear environments are investigated and compared to those of an integer order admittance controller. 


\subsection{Related Work}

Ensuring stability of pHRI systems is challenging due to the presence of human in the control loop. The coupled stability analysis that involves the human operator coupled with the rest of the system is not trivial, since human dynamics display nonlinear, time and configuration-dependent characteristics and hence is hard to model and analyze. Interaction with an environment makes this analysis even more challenging, as the impedance of the contact environment is also likely to be nonlinear and even uncertain. In such cases, the robot interacts with a combined impedance of human arm and environment; hence, a comprehensive model for their dynamics is required to conduct stability analysis. It is highly challenging to develop such a model that can also be integrated with existing analysis methods.

Modeling the dynamics of human arm and environment as linear second order mass-spring-damper systems is a commonly used simplifying assumption that allows one to identify basic factors effecting the coupled stability. As it has been observed in the literature, the human arm and environment model, specifically the stiffness components in those models, have a direct effect on the coupled stability of admittance controllers [4], [5]. In particular, the rootlocus technique has been used by Tsumugiwa et al. [5] to determine the coupled stability of a control system designed for a pHRI task and it has been shown that the environment stiffness is the most dominant factor contributing to the instability of the system. Duchaine et al. [6] have estimated the human arm stiffness experimentally and utilized this information in Lyapunov stability analysis to determine the critical damping required for the closed-loop system to be stable. This method relies on the maximum arm stiffness identified offline; hence, neglects the time-varying nature of human arm dynamics. In contrast, in [7], human arm model has been estimated on-line using EMG sensors and controller gains are adjusted on-the-fly based on the estimated arm stiffness to ensure coupled stability of the system. Instead, in [8], human arm stiffness is estimated indirectly by measuring the grasping force applied by human. It is assumed that the tight grasp by the human results in an increase in the stiffness reflected to robot, and the controller gains are adjusted accordingly. However, this method requires design of a gain scheduler and additional handles equipped with pressure sensors for the implementation.

The coupled stability analysis of the pHRI systems for a large class of human and environment models can also be conducted using the passivity framework [9], [10]. This approach assumes that human operator behaves as a passive network element in the closed-loop system and does not intentionally generate energy to destabilize the system. Therefore, if the rest of the control system can be guaranteed to be strictly passive, one can argue that the closed-loop system is also passive, and the coupled stability can be ensured. In this approach, stability is guaranteed for large ranges of the human arm/environmental impedances. However, satisfying passivity condition brings conservative constraints on the controller gains [10], [11], which can degrade the task performance in $\mathrm{pHRI}$.

As an alternative, Hannaford and Ryu [12] proposed time-domain passivity approach, based on a passivity ob- server (PO) and a passivity controller (PC). Instead of using fixed controller gains throughout the interaction, PO estimates energy flow in and out of a system, and if an active behavior is detected, PC acts as an adaptive dissipative element to absorb the excessive energy measured by the PO. In [13], this approach has been extended to teleoperation and its effectiveness has been experimentally validated. Later, a more general framework based on this approach has been proposed for larger class of control systems [14]. Utilizing time domain passivity framework helps relax the conservativeness of the frequency domain passivity framework and promise stable human-robot interactions in the absence of human and environment models. However, this method requires estimation of exchanged energy through sensor measurements, which may prove challenging due to sampling, noise, and quantization. Furthermore, abrupt engagement of PC may disturb the quality of interaction.

Satisfying passivity throughout the interaction ensures that robot behavior is never active (i.e. energy is not generated), so that stability can be guaranteed for a large range of human/environment impedances. The conservativeness of the passivity frameworks can further be relaxed by taking advantage of partial knowledge of the model of human and environment. For instance, even though the dynamics of human arm changes over time, human arm impedance varies in a relatively limited range [15], [16]. Along these lines, Haddadi and Hashtrudi-Zaad [17] relaxed the passivity constraints when the the bounds of the environmental/human arm impedances are known.

Given that non-passive (i.e. active) systems are not necessarily unstable [18], Buerger and Hogan [19] proposed a complementary stability approach to ensure robust stability without the need for passivity. In this approach, the goal is to design a robust controller that can maintain stability for a bounded range of human/environment impedances. Due to the complex interaction of the control parameters with the coupled human-robot system, analytical methods prove to be prohibitive for complementary stability; hence, this approach relies upon computational techniques.

\subsection{Contributions}

Earlier studies in pHRI rely on integer order admittance (or impedance) controllers (IOAC). In our recent study [20], we have provided evidence that a fractional order admittance controller (FOAC) can offer better stability characteristics than IOAC for pHRI. This paper significantly extends our initial results by providing more comprehensive theoretical and empirical comparisons of FOAC and IOAC. In particular, in terms of theoretical analysis, we extend our stability maps to cover multiple differentiation orders, introduce the concept of impedance matching to enable fair comparisons between FOAC and IOAC, and present stability robustness and transparency analyses of both controllers. We also extend our empirical investigations by providing results of a needle insertion experiment that involves a more complex nonlinear environment.

FOAC introduces an extra controller parameter, the integration order, that helps regulate the dynamics of the interaction between human and the robot, bringing more flexibility to the controller design. FOAC is advantageous 
as it enables effective control of the frequency dependent response, while still relying on the powerful analysis tools provided by linear systems theory. For instance, FOAC allows for effective damping to increase with frequency, providing further robustness at relatively high frequencies, while not significantly interfering with the quality of the interaction at low frequencies where intentional control takes place.

The rest of this article is organized as follows: Section 2 presents preliminaries and the control architectures used in this study. Stability, transparency, impedance matching, and stability robustness analyses are reported in Section 3. Two different human subject experiments conducted to assess the task performance during interactions under FOAC and IOAC are reported in Sections 4 and 5, respectively. Conclusions and future research directions are provided in Section 6.

\section{PRELIMINARIES}

\subsection{Fractional Order Control}

Fractional order calculus, a generalization of well-known integer order calculus, allows differentiation and integration of arbitrary orders. Fractional order calculus has been successfully utilized in many application areas, ranging from modeling of viscoelastic materials [21] to motion control of robotic systems. Impedance controllers based on fractional order calculus have been proposed and analysed in [22], [23] for haptic rendering.

Calculating a fractional order derivative/integral (differintegral) is not trivial. There exist multiple mathematically well-founded definitions for differintegration and one has to choose the appropriate definition based on the application area. In this study, the Grunwald-Letnikov definition is used for calculation of fractional order differintegrals, since this definition enables easier implementation in discrete time domain [24].

The Grunwald-Letnikov definition for fractional order differintegral is given as

$$
D^{\alpha} y(t)=\lim _{h \rightarrow 0} \frac{1}{h^{\alpha}} \sum_{i=0}^{(t-a) / h}{ }^{\alpha} C_{i} y(t-i h)
$$

where $\alpha$ is the order of the differintegral, $h$ is the step size of the calculation, $a$ is the window length, and ${ }^{\alpha} C_{i}$ can be evaluated recursively from

$$
{ }^{\alpha} C_{0}=1, \quad{ }^{\alpha} C_{i}={ }^{\alpha} C_{i-1}\left(1-\frac{\alpha+1}{i}\right), \quad i=1,2, \ldots
$$

Fractional order differintegral is a linear operator; hence, fractional order systems/controllers can be designed as linear time-invariant (LTI) systems and analysed using familiar methods. However, fractional order differintegral is a nonlocal operator, that is, it has a memory and relies on the whole history. In real-time implementation, relying upon the short memory principle, the summation is approximated using a finite horizon into the recent past, as the earlier values are known to have a rapidly decaying influence on the current solution.

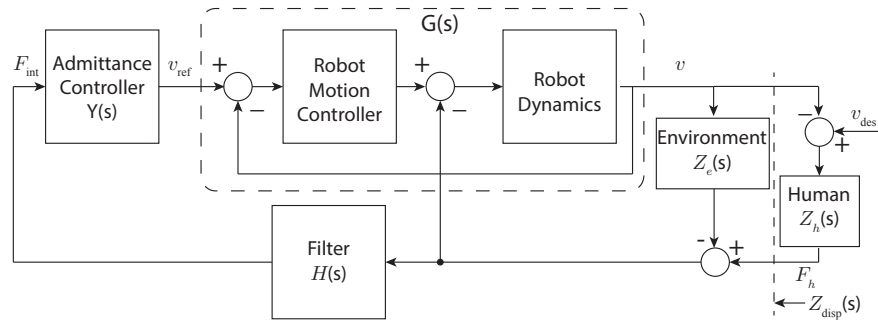

Fig. 1. Control architecture of the pHRI system

\subsection{Characterization of the Robot}

In our experiments, subjects interact with a UR5 robot (Universal Robots Inc.) to perform pHRI tasks. Since the dynamical model of the robot is not provided by the manufacturer, it is obtained experimentally along the horizontal axis for a specific joint configuration. That is, a transfer function $G(s)$ of the robot is estimated between recorded $V(s)$ and commanded (reference) $V_{\text {ref }}(s)$ velocities of the end-effector, where

$$
G(s)=\frac{1.45 \mathrm{e}-6 s^{3}-0.75 s^{2}+3.92 e 5 s+5.48 e 7}{s^{4}+190.9 s^{3}+2.73 e 4 s^{2}+1.72 e 6 s+5.55 e 7}
$$

\subsection{Control Architecture}

Fig. 1 shows the admittance control architecture used in this study. In this architecture, we assume that there are physical interactions among human, robot, and environment. Human applies force, $F_{h}$, to the robot to move it with a desired velocity, $v_{\text {des }}$ and interact with the environment. The resultant interaction force is measured by a force sensor attached to the end-effector of the robot. This measurement is filtered by a low pass filter $H(s)$, and the filtered interaction force, $F_{\text {int }}$, is then sent to the admittance controller $Y(s)$. The controller generates the corresponding reference velocity $v_{\text {ref }}$. Then, robot's motion controller transmits sufficient torque to its joints in order to closely achieve the reference velocity. The robot's motion controller is robust to the environmental forces acting on its end-effector and it is assumed that the motion of the robot is not significantly affected by these forces. The transfer function of the closed loop system is given by

$$
T(s)=\frac{V(s)}{F_{\text {int }}(s)}=\frac{G(s) Y(s)}{1+G(s) Y(s) H(s) Z_{\text {eq }}(s)}
$$

Human and environment are assumed to be coupled, and $Z_{\text {eq }}=Z_{h}+Z_{e}$ represents the equivalent impedance, where $Z_{h}$ and $Z_{e}$ represent human and environment impedances, respectively.

Using the control architecture given in Fig. 1, we compare IOAC and FOAC in terms of stability and closed loop impedance characteristics.

\subsubsection{Integer order admittance controller}

IOAC has been already used in earlier pHRI studies in the following form:

$$
Y(s)=\frac{1}{Z_{\mathrm{IOAC}}}=\frac{1}{m_{I} s+b_{I}}
$$

where $Z_{\mathrm{IOAC}}(s)$ represents the denominator of the IOAC, and $m_{I}$ and $b_{I}$ are the admittance controller parameters, corresponding to mass and damping, respectively. 


\subsubsection{Fractional order admittance controller}

FOAC is defined as

$$
Y(s)=\frac{1}{Z_{\mathrm{FOAC}}(s)}=\frac{1}{m_{F} s^{\alpha}+b_{F}}
$$

where $\alpha$ corresponds to the fractional order of the integrator. Here, $Z_{\mathrm{FOAC}}(s)$ stands for the denominator of the FOAC, and $m_{F}$ and $b_{F}$ are the admittance controller parameters. In this study, the range of the integration order is kept as $0<\alpha \leq 1$. Thanks to the analytical nature of fractional order differintegration, as the integration order is decreased gradually from 1 to 0 , the inertial energy storage characteristics of the controller decay and energy dissipation characteristics dominate. For $\alpha=0$, the controller acts like a pure damper. On the other hand, when the integration order is $\alpha=1$, FOAC becomes IOAC. Therefore, the effective mass and damping [23] supplied by the controller to the system change depending on the integration order $\alpha$, while dissipative effects are present throughout the range $0<\alpha<1$. To demonstrate the effect of integration order on dynamical response of FOAC, consider $Z_{\mathrm{FOAC}}$ expressed in the frequency domain

$$
\begin{aligned}
Z_{\mathrm{FOAC}}(j \omega) & =m_{F}(j \omega)^{\alpha}+b_{F} \\
& =b_{F}+m_{F} \omega^{\alpha} \cos \left(\frac{\alpha \pi}{2}\right)+j m_{F} \omega^{\alpha} \sin \left(\frac{\alpha \pi}{2}\right)
\end{aligned}
$$

In this equation, the effective mass and damping provided by FOAC are $m_{F} \omega^{\alpha-1} \sin \left(\frac{\alpha \pi}{2}\right)$ and $b_{F}+m_{F} \omega^{\alpha} \cos \left(\frac{\alpha \pi}{2}\right)$, respectively, whereas these values are simply $m_{I}$ and $b_{I}$ for IOAC. Hence, unlike in the integer order case, the effective damping in FOAC is frequency-dependent, and can be adjusted by altering the integration order $\alpha$. This property of FOAC is of interest for pHRI tasks, as it can improve stability robustness of interactions in tasks involving contacts with environment.

\section{AnAlysis of the Controllers}

This section investigates the coupled stability of the proposed FOAC and compares it with IOAC. Furthermore, the transparency of these systems are also investigated using the effective impedance analysis [25], [26].

In these analyses, human impedance is modeled as a second order LTI system as

$$
Z_{h}(s)=\frac{m_{h} s^{2}+b_{h} s+k_{h}}{s}
$$

where $m_{h}, b_{h}, k_{h}$ represent human arm mass, damping, and stiffness, respectively. Moreover, contact environment is modelled as a spring with stiffness of $k_{e}$, where environment impedance is $Z_{e}=k_{e} / s$. In our analyses, we combine human arm and environment impedance and investigate the stability robustness to variations in the stiffness component of the equivalent impedance, which is the main factor affecting the stability as suggested in earlier studies [4], [5]. The equivalent impedance used in our study is $Z_{\mathrm{eq}}=\frac{m_{h} s^{2}+b_{h} s+k_{\mathrm{eq}}}{s}$, where equivalent stiffness becomes $k_{\mathrm{eq}}=k_{h}+k_{e}$.

\subsection{Stability}

For safe pHRI applications, it is imperative to guarantee the coupled stability of the closed-loop system. Stability analysis of interaction control is challenging, as the standard tools used for servo control cannot be applied. In particular, as noted in [19], two fundamental differences exist for interaction control systems: the closed-loop stability and performance cannot be predicted and characterized using the open-loop transfer function, and the controller does not predictably affect the stability and performance of the coupled system. Consequently, there exists no obvious way to analytically study stability and performance of such systems, while computational approaches have been shown to provide promising results [19].

Along these lines, we compare performance of FOAC with IOAC through computational stability and transparency analyses of these controllers. It is important to note that fractional order nature of the control systems makes any form of analytical analysis even more challenging.

The stability characteristics of the coupled system is analyzed for different controller parameters using stability maps. A stability map is a graphical representation of the controller parameters for which the resulting closed-loop system becomes stable for a range of environment/human arm impedances. The stability analysis is performed by numerically studying the location of the closed-loop poles of the system. The search for a stable set of controller parameters is relatively straightforward when the admittance controller is of integer order. However, when a fractional order element is used in the control loop, the resulting characteristic equation no longer takes a polynomial form; hence, the stability analysis becomes more involved.

For fractional order systems that are of commensurate order, checking the stability of the system with a nonpolynomial characteristic equation can be accomplished by performing a transformation from the fractional order domain to the integer order domain. According to Matignon's theorem [24], [27], the resulting integer order system possesses equivalent stability characteristics as the fractional order system.

The stability maps for FOAC ( $\alpha=0.4$ and 0.7$)$, and IOAC $(\alpha=1)$ are constructed to investigate the effect of integration order $\alpha$ on stability robustness to the changes in equivalent stiffness $k_{\text {eq }}$ of the system. The range for the values of $k_{\text {eq }}$ is set to be comparable to the maximum value of human arm stiffness reported in [15], [16]. In these analyses, it is assumed that human always maintains his/her contact with the end-effector of the robot. In such a condition, mass and damping levels in human arm model would not change significantly, where the arm stiffness may change due to the co-contraction of muscles. Hence, the robustness of the proposed controller is investigated for changes in equivalent stiffness, as it has been reported that change in stiffness of human arm and/or environment significantly contributes to the instabilities observed in pHRI [4], [5]. In particular, the stability analyses are conducted for a range of equivalent stiffness values, while typical values of $b_{h}=15 \mathrm{Ns} / \mathrm{m}$ and $m_{h}=1.5 \mathrm{~kg}$ are used for the reflected arm damping and mass, respectively.

Fig. 2a depicts the stability map for IOAC for $k_{\mathrm{eq}} \in$ 


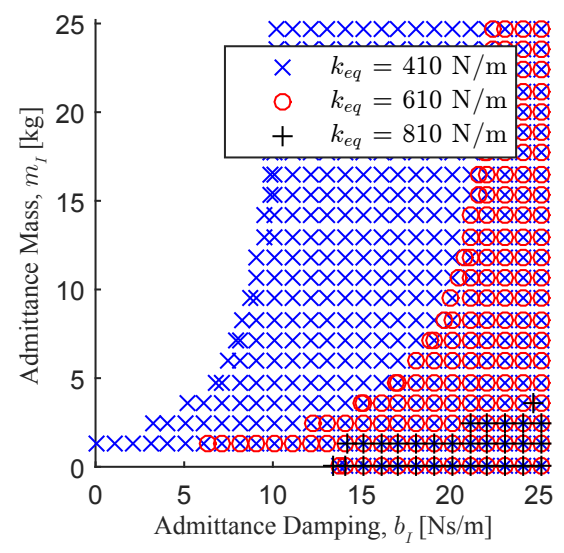

(a) $\alpha=1$

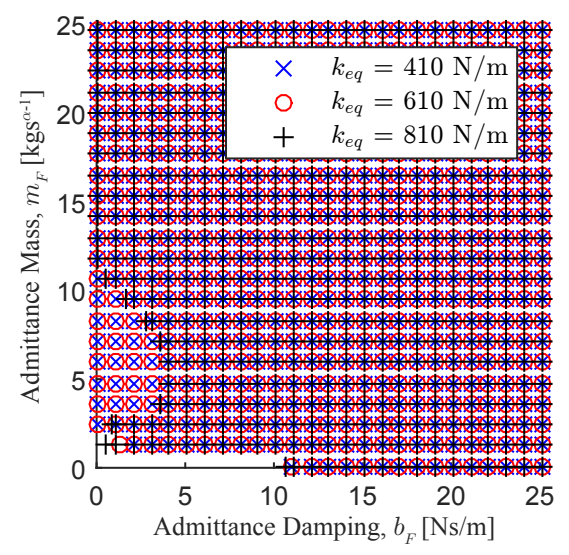

(b) $\alpha=0.7$

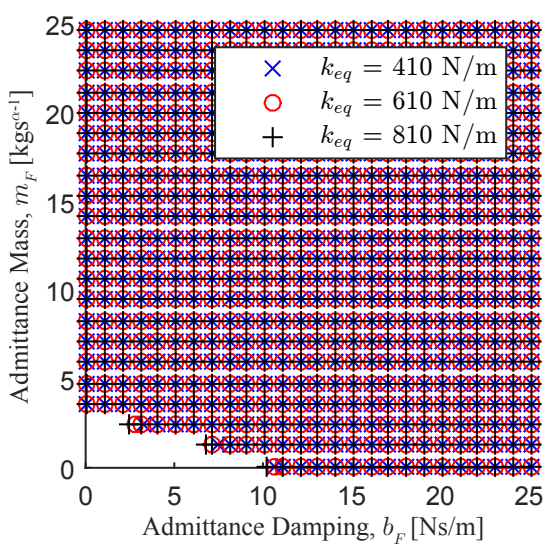

(c) $\alpha=0.4$

Fig. 2. Stability maps of pHRI system for stiffness range $k_{\text {eq }} \in\{410-810\} \mathrm{N} / \mathrm{m}$ under (a) IOAC $\alpha=1$, (b) FOAC $\alpha=0.7$, and (c) FOAC $\alpha=0.4$.

$\{410-810 \mathrm{~N} / \mathrm{m}\}$, where a relatively large range of values are utilized for equivalent stiffness since the stiffness of human arm is assumed to be coupled with that of the environment. From these plots, the destabilizing nature of the equivalent stiffness can be observed; as the equivalent stiffness increases, the area for which the closed-loop system is stable gets smaller. Moreover, it can be seen that higher admittance damping is required to stabilize the system for higher values of equivalent stiffness, which is consistent with the findings reported in [4], [5].

Similar analysis is conducted for FOAC and the resulting stability maps for $\alpha=0.7$ and $\alpha=0.4$ are presented in Figs. $2 \mathrm{~b}$ and 2c, respectively. It can be observed from these plots that the change in the stability regions of FOAC for varying equivalent stiffness levels is much less compared to that of IOAC. In addition, it can be observed that robustness to variations in equivalent stiffness increases as integration order $\alpha$ decreases (Figs. 2b and 2c).

These results indicate that, under the assumptions of a second order LTI model for human arm and contact interaction with a relatively stiff environment modelled as a linear spring, parameters of FOAC can exhibit significantly higher levels of robustness to the changes in the environment/human arm stiffness values.

\subsection{Transparency}

We computationally compare the transparency of pHRI systems under IOAC and FOAC through studying their effective closed loop impedances. For the system in Fig. 1, the closed loop impedance displayed to human $Z_{\text {disp }}(s)$ is

$$
Z_{\text {disp }}(s)=\frac{F_{h}(s)}{V(s)}=\frac{1+G(s) Y(s) H(s) Z_{e}(s)}{G(s) Y(s) H(s)}
$$

The impedance reflected to human can then be decomposed into effective mass $\left(E M(\omega)=\omega^{-1} \Im^{+}\left\{Z_{\text {disp }}(j \omega)\right\}\right)$, effective damping $\left(E D(\omega)=\Re^{+}\left\{Z_{\text {disp }}(j \omega)\right\}\right)$, and effective stiffness $\left(E S(\omega)=\omega \Im^{-}\left\{Z_{\text {disp }}(j \omega)\right\}\right)$ components, as suggested in [25], [26], where $Z_{\text {disp }}(j \omega)$ is the frequency response of the closed loop impedance transfer function.

For a comparison of IOAC with FOAC, a stiffness value of $k_{e}=610 \mathrm{~N} / \mathrm{m}$ is selected for the environment. The admittance controller parameters are set as $m_{F}=\{5,20\}$ $\mathrm{kgs}^{\alpha-1}, m_{I}=\{5,20\} \mathrm{kg}$, and $b_{F}=b_{I}=25 \mathrm{Ns} / \mathrm{m}$. These values are chosen from the stable region in the stability maps in Fig. 2. The effective mass, damping, and stiffness for these systems as a function of frequency are depicted in Fig. 3a-c. It can be observed from these plots that the effective mass stays close to zero up to the natural frequency $\left(\omega_{n}\right)$, since effective stiffness dominates the dynamical behavior within that range. When $\omega>\omega_{n}$, the effective mass for IOAC is higher than that of FOAC. The effective stiffness is higher under FOAC than that of IOAC for $\omega<\omega_{n}$. Afterwards, it becomes close to zero as the effective mass dominates the behavior of the system for $\omega>\omega_{n}$. Natural frequency of the system under FOAC is higher than that of IOAC, since higher effective stiffness and lower effective mass are rendered under FOAC. This behaviour can also be observed by inspecting the magnitude and phase plots (Figs. 3d and 3e) of the closed loop system.

Effective damping analysis in Fig. $3 \mathrm{c}$ reveals that the effective damping supplied by FOAC remains high for a larger range of frequencies compared to IOAC and displays an increase with frequency almost up to the natural frequency of the system. This behaviour is beneficial as higher effective damping at higher frequencies contributes to stability robustness, while relatively lower effective damping at lower frequencies may lead to higher task performance due to better transparency.

\subsection{Impedance Matching}

Providing the range of controller parameters under which the closed loop system stays stable, stability maps are useful to study the stability robustness of a system under uncertainties. However, stability maps cannot be employed to directly compare controllers of different integration orders, as the control parameters for each integration order have different physical characteristics and different units. Furthermore, given the frequency dependent nature of FOAC, a direct analytical mapping between IOAC and FOAC parameters can only be provided at a particular frequency.

To enable a comparison between IOAC and FOAC parameters, we propose impedance matching to map the parameters of FOAC to the parameters of an equivalent IOAC at a given frequency of interest. In particular, given a frequency $\left(\omega_{0}\right)$, the closed loop impedance under FOAC can be matched to that of IOAC using (9). Since $Z_{e}, H$, and $G$ are 


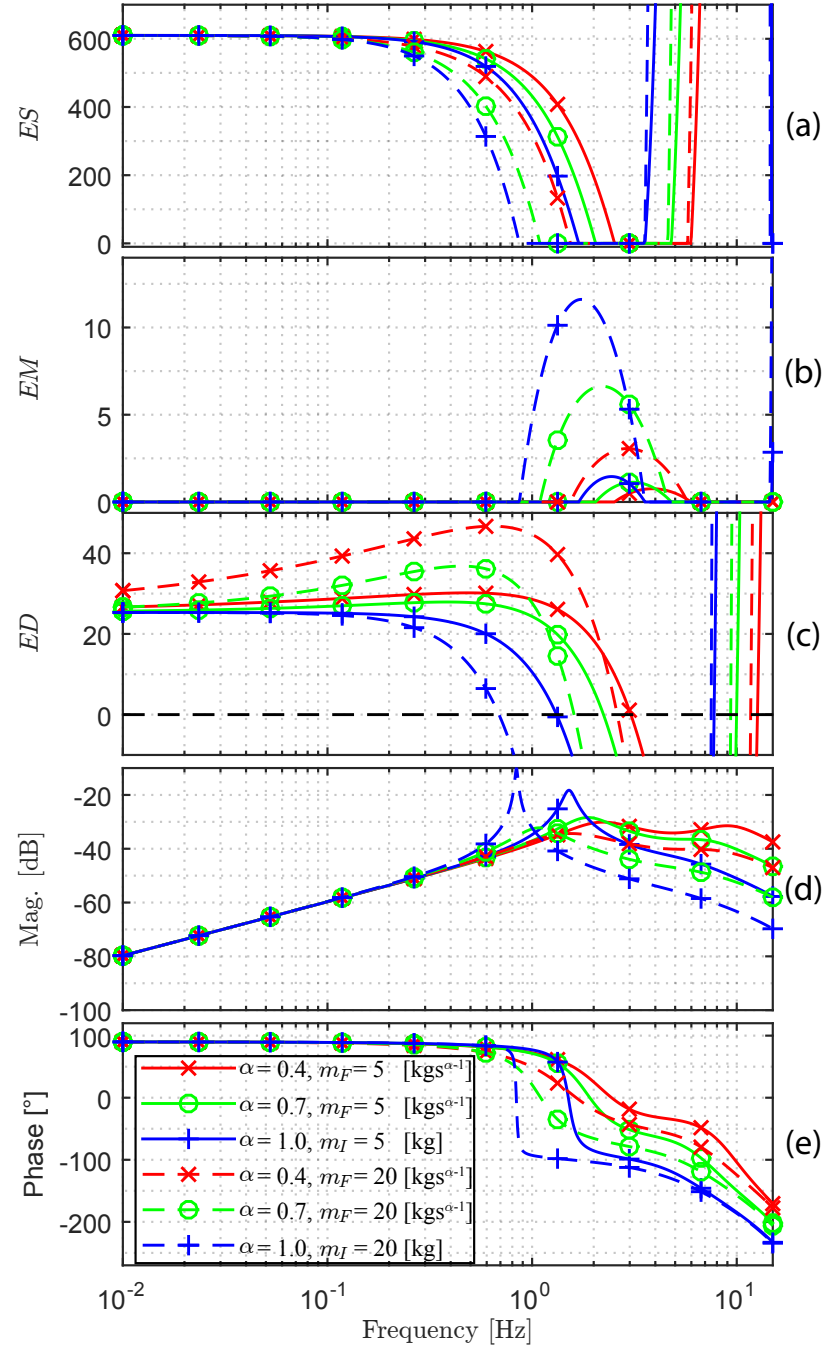

Fig. 3. Effective stiffness (a), effective mass (b), and effective damping (c) of the impedance transfer function. Magnitude (d) and phase (e) plots of the closed loop transfer function.

the same for both controllers in (9), equating the controller admittance transfer functions $Y$ becomes sufficient for the closed loop impedance matching at a particular frequency $\omega_{0}$ as follows

$$
Y_{I}\left(j \omega_{0}\right)=Y_{F}\left(j \omega_{0}\right) \Longrightarrow \frac{1}{m_{I} j \omega_{0}+b_{I}}=\frac{1}{m_{F}\left(j \omega_{0}\right)^{\alpha}+b_{F}}
$$

It can be shown that the above complex equality is satisfied, when the following equivalence relations hold among the controller parameters

$$
\begin{aligned}
b_{I} & =b_{F}+m_{F} \omega_{0}^{\alpha} \cos \left(\frac{\pi \alpha}{2}\right) \\
m_{I} & =m_{F} \omega_{0}^{\alpha-1} \sin \left(\frac{\pi \alpha}{2}\right)
\end{aligned}
$$

Under the impedance matching, these equations provide a direct mapping among parameters of FOAC and IOAC, at $\omega_{0}$. Note that, while FOAC is designed as a linear timeinvariant (LTI) system and analyzed using familiar methods, FOAC provides frequency dependent admittance controller parameters. One can observe from these equations that, for $\alpha<1$, the mapped damping parameter of FOAC increases, and the mapped mass parameter of FOAC decreases, as $\omega_{0}$ increases. However, the controller parameters for an IOAC are independent of $\omega$. Frequency dependence of FOAC is beneficial as low values of mapped damping parameter at low frequencies can improve transparency, while high values of mapped damping parameter at high frequencies is beneficial for stability robustness. Similarly, decrease in mapped mass parameter as frequency increases may provide improved transparency. These effects are consistent with the observations due to the effective impedance analysis of the closed loop system in the previous subsection.

\subsection{Stability Robustness}

The phase lag added by a controller can be studied as an indicator of the stability robustness for LTI systems. Let $\tau_{F}=m_{F} / b_{F}$ and $\tau_{I}=m_{I} / b_{I}$ be defined as the time constants for FOAC and IOAC, respectively. The phase contributions of IOAC and FOAC under impedance matching at a given frequency $\omega_{0}$ can be derived as

$$
\begin{aligned}
& \phi_{I}=-\tan ^{-1}\left(\tau_{I} \omega\right)=-\tan ^{-1}\left(\frac{\tau_{F} \omega_{0}^{\alpha-1} \sin \left(\frac{\pi \alpha}{2}\right)}{1+\tau_{F} \omega_{0}^{\alpha} \cos \left(\frac{\pi \alpha}{2}\right)} \omega\right) \\
& \phi_{F}=-\tan ^{-1}\left(\frac{\tau_{F} \omega^{\alpha} \sin \left(\frac{\pi \alpha}{2}\right)}{1+\tau_{F} \omega^{\alpha} \cos \left(\frac{\pi \alpha}{2}\right)}\right)
\end{aligned}
$$

Studying the case when $\alpha<1$, as $\omega \rightarrow 0, \phi_{I}$ and $\phi_{F}$ both go to zero, whereas as $\omega \rightarrow \infty, \phi_{I} \rightarrow \pi / 2$, while $\phi_{F} \rightarrow \pi \alpha / 2$. The values of $\phi_{I}$ and $\phi_{F}$ are close to each other for $\omega \leq \omega_{0}$. On the other hand, $\phi_{I}$ increases rapidly as a function of $\omega$ especially for $\omega>\omega_{0}$, whereas $\phi_{F}$ increases with a slower rate. Thus, for frequencies, especially beyond $\omega_{0}$, IOAC always adds more phase to the loop transfer function than FOAC does, which means that the phase lag under FOAC is lower.

In general, the phase margin alone is not a sufficient indicator of the stability robustness of a system, while the vector margin, defined as the inverse of the maximum magnitude of loop sensitivity function $S$ [28], can be studied for more conclusive results. The sensitivity function $S$ for the system can be defined as

$$
S=\frac{1}{1+L}=\frac{1}{1+G Y H Z_{\mathrm{eq}}}
$$

where $L$ denotes the loop transfer function. From this equation, one can note that a larger loop gain reduces the magnitude of the sensitivity function and leads to a more robust system. For a given $G H Z_{\text {eq, }}$ one can compare the controller gains of IOAC and FOAC to investigate which one is more robust under impedance matching at $\omega_{0}$. In the equations below, $Z_{\mathrm{FOAC}}$ and $Z_{\mathrm{IOAC}}$ stand for the denominator of FOAC and IOAC, respectively.

$$
\begin{aligned}
\left|Z_{\mathrm{FOAC}}\right|^{2} & =m_{F}^{2} \omega^{2 \alpha}+b_{F}^{2}+2 m_{F} b_{F} \cos \left(\frac{\pi \alpha}{2}\right) \omega^{\alpha} \\
\left|Z_{\mathrm{IOAC}}\right|^{2} & =m_{I}^{2} \omega^{2}+b_{I}^{2} \\
& =m_{F}^{2} \omega_{0}^{2 \alpha-2} \sin ^{2}\left(\frac{\pi \alpha}{2}\right) \omega^{2}+b_{F}^{2} \\
& +m_{F}^{2} \omega_{0}^{2 \alpha} \cos ^{2}\left(\frac{\pi \alpha}{2}\right)+2 m_{F} b_{F} \omega_{0}^{\alpha} \cos \left(\frac{\pi \alpha}{2}\right)
\end{aligned}
$$

As $\omega \rightarrow 0,\left|Z_{\mathrm{FOAC}}\right|^{2} \rightarrow b_{F}^{2}$, whereas $\left|Z_{\mathrm{IOAC}}\right|^{2} \rightarrow b_{F}^{2}+$ $m_{F}^{2} \omega_{0}^{2 \alpha} \cos ^{2}(\pi \alpha / 2)+2 m_{F} b_{F} \omega_{0}^{\alpha} \cos (\pi \alpha / 2)$. As $\omega \rightarrow \infty$, 
$\left|Z_{\mathrm{IOAC}}\right|^{2}$ increases with $\omega^{2}$ while $\left|Z_{\mathrm{FOAC}}\right|^{2}$ increases with $\omega^{2 \alpha}$, which has a slower rate when the differentiation order $\alpha<1$.

Consequently, given that the magnitude of the sensitivity function is lower under FOAC, the vector margin of FOAC is larger than that of an IOAC with a matched impedance at a given frequency $\omega_{0}$. These results are consistent with the stability map analysis presented in Section 3.1.

\section{A Controlled PHRI Experiment: Continu- OUS CONTACT WITH A SPRING}

In pHRI, maximizing task performance, while maintaining stability is desired. The effective impedance analysis shows that high effective damping under FOAC can be sustained for a larger range of frequencies, which is desirable for stable interactions. This feature of FOAC can improve human performance in pHRI tasks involving especially contact interactions with environment.

A controlled human subject experiment was designed to further investigate this frequency-dependent damping feature of FOAC, and effect of controller type (i.e. FOAC vs IOAC) and admittance parameters $m_{F}$ and $m_{I}$ on performance in a pHRI task. The task involved contact interactions with a linear spring, where spring represented a relatively stiff environment.

\subsection{Experimental Procedure}

In our experimental setup, a spring $\left(k_{e}=610 \mathrm{~N} / \mathrm{m}\right)$ is connected between a fixed support and the end-effector of UR5 robot, as shown in Fig. 4a. The subject grasps the handle between the spring and the robot to guide the end-effector and compress the spring. The spring simply represents the environment in which the robot guided by a human operator is in continuous contact, as in drilling a wall or inserting a needle into soft tissue. In the experiments, the subjects are asked to compress the spring up to a certain position (ramp phase, Fig. 4b), and then, hold it at that position for 10 seconds (hold phase, Fig. 4c). The details of the experimental procedure is available in our earlier publication [20].

We compared subjects' performance under IOAC $(\alpha=$ $1)$ and FOAC $(\alpha=0.7)$. Since parameters $m_{F}$ and $m_{I}$ are the coefficients in front of the integrator in admittance controller, we also investigated their effect on performance for low (i.e. LAM, $m_{F}=5 \mathrm{kgs}^{\alpha-1}, m_{I}=5 \mathrm{~kg}$ ) and high (i.e. HAM, $m_{F}=20 \mathrm{kgs}^{\alpha-1}, m_{I}=20 \mathrm{~kg}$ ) values. On the other hand, the admittance damping $\left(b_{F}=b_{I}=25\right.$ $\mathrm{Ns} / \mathrm{m}$ ) was kept constant throughout the experiment, since it does not have a direct relation with the integrator. These values were chosen by considering the stable regions of the stability maps given in Fig. 2. Hence, four different conditions (FOAC-LAM, IOAC-LAM, FOAC-HAM, IOACHAM) were tested in this experiment. Each condition was repeated 9 times. Thus, there were $36(4 \times 9)$ trials in the experiment, which were displayed to the subjects in random order, while the order was same for each subject. Prior to the actual experiment, each subject was given a training session to get her/him familiar with the setup. 10 subjects ( 5 males and 5 females, average age $=28 \pm 5$ ) participated in this

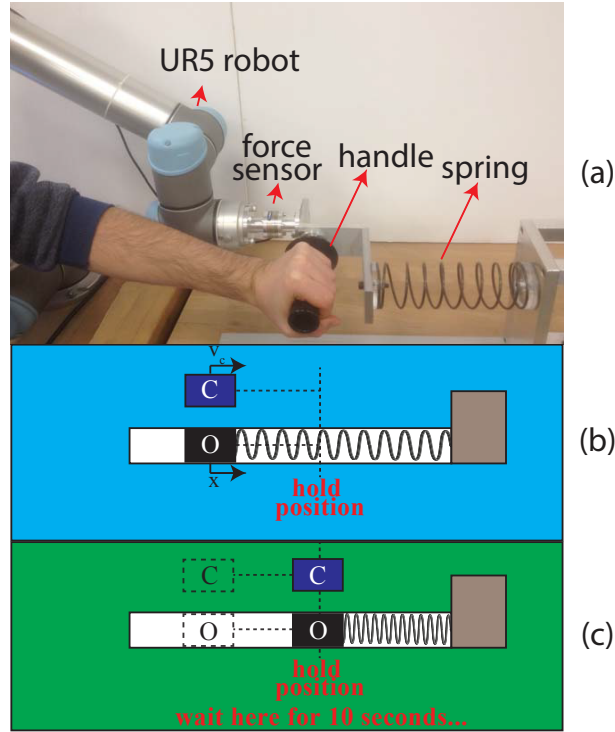

Fig. 4. The experimental setup and visual feedback provided to the subjects during the experiments.

experiment. The subjects gave informed consent about their participation in the experiment.

\subsection{Data Collection and Metrics}

During the experiment, the robot constrains the motion of the subjects along a horizontal line while they compress the spring. The force $F_{h}$ applied by a subject is the sum of the force $F_{e}$ required for compressing the spring and generating the motion trajectory of the robot (i.e. interaction force $F_{\text {int }}$ ) $F_{h}=F_{e}+F_{\text {int }}$, where $F_{e}=k_{e} \Delta x(\Delta x$ is the amount of compression) is not influenced by the controller type or admittance parameters $m_{F}$ and $m_{I} . F_{\text {int }}$ is the interaction force measured by a force sensor (Mini40, ATI Inc.), filtered, and fed back to the admittance controller. The force applied by the subject $F_{h}$ and the interaction force $F_{\text {int }}$ are linearly dependent on each other, since $F_{e}$ depends on only the amount of compression, and is independent of viscous and inertial effects coming from the controller and the robot. Hence, by inspecting the interaction force alone, we can deduce how the force applied by the subject is influenced by the changes in admittance controller, and parameters $m_{F}$ and $m_{I}$

All the metrics evaluated in this section are based on this $F_{\text {int }}$ (the filtered value of the interaction force), unless otherwise is specified. We use average power ( $P^{\text {ave }}=$ $\left.1 /\left(t_{f}-t_{i}\right) \int_{t_{i}}^{t_{f}}\left|F_{\text {int }}(t) \cdot v(t)\right| d t\right)$, and average interaction force $\left(F_{\text {int }}^{\text {ave }}=1 /\left(t_{f}-t_{i}\right) \int_{t_{f}}^{t_{f}}\left|F_{\text {int }}(t)\right| d t\right)$ to quantify the interaction performance under different task conditions, where, $t_{i}$ and $t_{f}$ are the beginning and ending times of a phase (ramp or hold), respectively. We also inspect average dominant frequency of oscillations in interaction force $\left(\Omega_{F_{\text {int }}}=\right.$ $\left.1 / N \sum_{i=1}^{N} 1 /\left(2\left|t_{\max , i}-t_{\min , i}\right|\right)\right)$, where $t_{\max , i}\left(t_{\min , i}\right)$ represents the time at $i^{\text {th }}$ local maximum (minimum), and $N$ stands for the total number of consecutive local minima and maxima in interaction force $F_{\text {int }}$. The results are also verified by FFT analysis in the frequency domain. In addition, average amplitude of oscillations in interaction force $\left(A_{F_{\text {int }}}=1 / N \sum_{i=1}^{N}\left|F_{\max , i}-F_{\min , i}\right|\right)$ and end-effector position $\left(A_{P}=1 / N \sum_{i=1}^{N}\left|x_{\max , i}-x_{\min , i}\right|\right)$ are computed, 

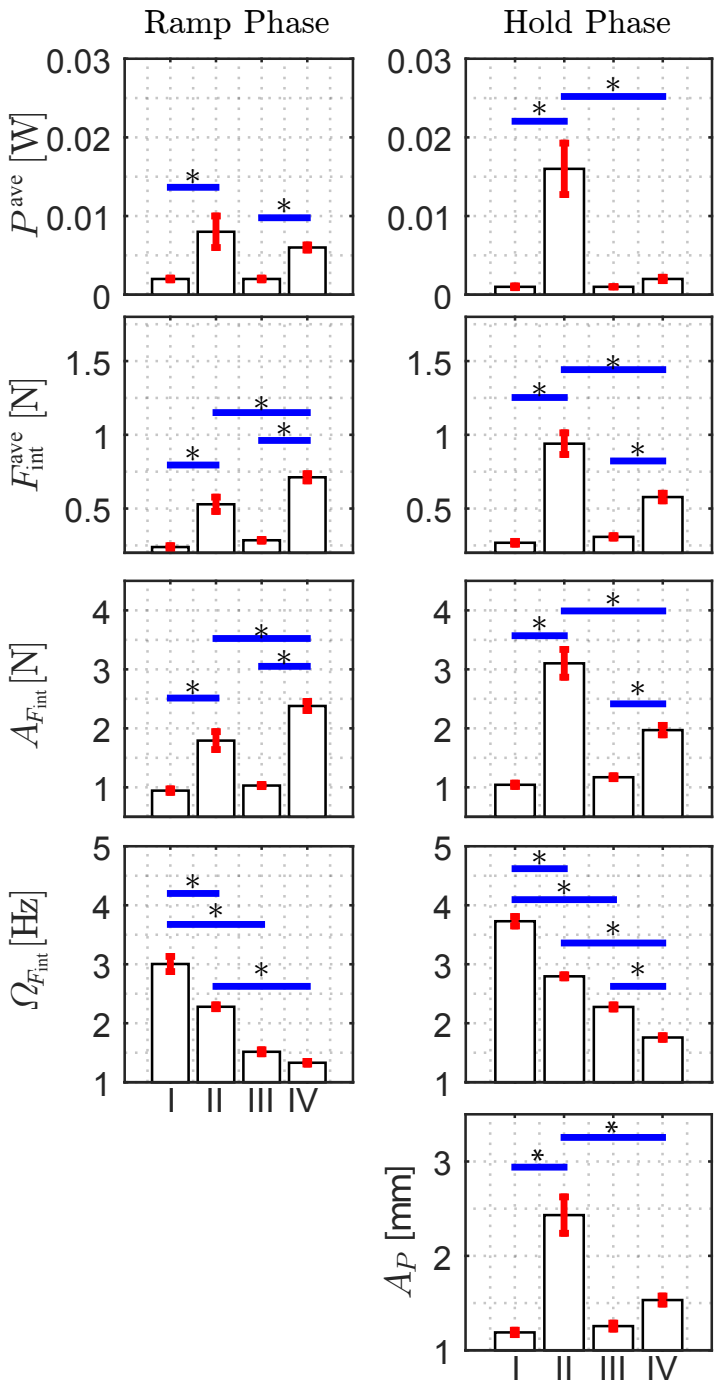

Fig. 5. The means and the standard errors of means of normalized performance metrics (I: FOAC-LAM, II: IOAC-LAM, III: FOAC-HAM, IV: IOAC-HAM. Horizontal bars with $*$ on top indicate statistical significance between results of the two corresponding conditions).

where $F_{\max , i}\left(F_{\min , i}\right)$ represents the $i^{\text {th }}$ local maximum (minimum) in interaction force $F_{\text {int }}$, and $x_{\max , i}\left(x_{\min , i}\right)$ indicates $i^{\text {th }}$ local maximum (minimum) in end-effector position $x$.

\subsection{Data Analysis}

For each subject, the performance metrics were calculated for the both phases (except $A_{P}$, which was evaluated for the hold phase only) of all trials and then normalized for the analysis (Fig. 5). We initially performed two-way ANOVA to investigate the statistical significance of these results. We observed that the factors (admittance parameters $m_{F}$ and $m_{I}$, and type of controller) had significant effects on the performance and there was an interaction between them. Further analysis was performed to evaluate the individual effects of parameters $m_{F}$ and $m_{I}$, and controller type on interaction performance. In all statistical analyses, a significance level of $p=0.005$ was used to test the null hypothesis.

\subsection{Results}

Fig. 5a demonstrates average power $P^{\text {ave }}$ during both phases. $P^{\text {ave }}$ was significantly lower under FOAC than that of IOAC during both phases when LAM was used. $P^{\text {ave }}$ was significantly lower under FOAC than that of IOAC during only the ramp phase when HAM was used. The change in admittance parameter $m_{F}$ did not affect $P^{\text {ave }}$ significantly under FOAC.

Fig. 5b illustrates average interaction force $F_{\text {int }}^{\text {ave }}$ during both phases. We observed a statistically significant effect of controller type on $F_{\text {int }}^{\text {ave }}$. Specifically, $F_{\text {int }}^{\text {ave }}$ was significantly lower under FOAC than that of IOAC during both phases. The effect of change in admittance parameter $m_{F}$ on $F_{\text {int }}^{\text {ave }}$ was not significant under FOAC.

Fig. $5 \mathrm{c}$ shows average amplitude of oscillations in interaction force $A_{F_{\text {int }}}$ during both phases. $A_{F_{\text {int }}}$ was significantly lower under FOAC than that of IOAC during both phases. The change in admittance parameter $m_{F}$ did not affect $A_{F_{\text {int }}}$ significantly under FOAC.

Average dominant frequency of oscillations in interaction force $\Omega_{F_{\text {int }}}$ is illustrated in Fig. $5 \mathrm{~d}$. $\Omega_{F_{\text {int }}}$ was significantly higher under FOAC than that of IOAC during both phases when LAM was used. $\Omega_{F_{\text {int }}}$ was significantly higher under FOAC than that of IOAC during the hold phase for HAM.

Average amplitude of oscillations in end-effector position $A_{P}$ during the hold phase is shown in Fig. 5e. $A_{P}$ was significantly lower under FOAC than that of IOAC when LAM was used. The effect of change in admittance parameter $m_{F}$ on $A_{P}$ was not significant under FOAC.

\subsection{Discussion}

We investigated the effect of FOAC on human performance in a pHRI task involving contact interactions with a relatively stiff environment; compressing a spring with a certain rate (ramp phase) and holding it there for 10 seconds (hold phase). Due to the frequency-dependent nature of damping in FOAC, the oscillations in the interaction force were better absorbed in both phases of the task (Fig. 5c).

For FOAC, the higher admittance parameter $m_{F}$ did not result in significant increase in $F_{\text {int }}^{\text {ave }}, P^{\text {ave }}$ and $A_{F_{\text {int }}}$, while higher $m_{F}$ resulted in significantly lower average frequency $\Omega_{F_{\text {int }}}$ for force oscillations. These results indicate that frequency dependent behavior of effective damping in the fractional order case is the main factor in achieving the desired level of dissipation for the task at hand. The main effect of higher $m_{F}$ and $m_{I}$ is to increase the effective mass of the system, and to decrease the system natural frequency.

As a downside of FOAC, frequency of oscillations in force increased slightly (Fig. 5d) due to lower effective mass rendered by FOAC (Fig. 3b). This increase may not be desirable during contact interactions. However, amplitudes of oscillations in force and end-effector position were significantly lower than those of IOAC, since FOAC supplied more effective damping to the system (Figs. 5c, and 5e). Moreover, average power, average interaction force, and average amplitude of oscillations in interaction force were all significantly lower under FOAC (Figs. 5a, 5b, and 5c).

\section{Needle Insertion Experiment}

The results of the experiment reported in Section 4.4 shows that FOAC can improve performance in tasks involving contact interactions. In that controlled experiment, the task 


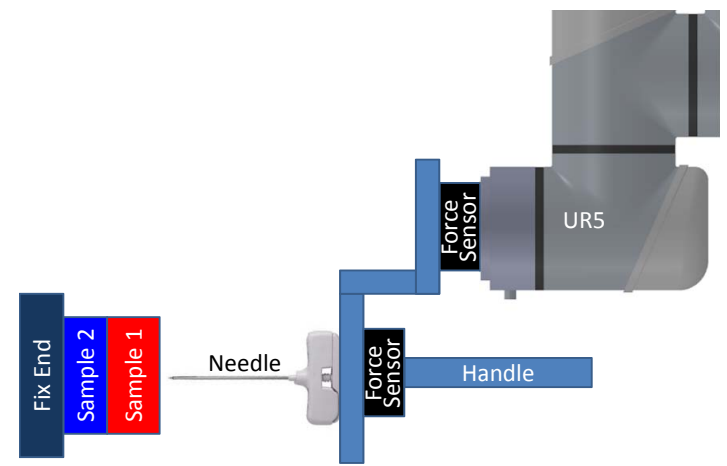

Fig. 6. Experimental setup for needle insertion experiments.

involved an interaction with a linear spring, which is sufficient for exploring the potential advantages of FOAC over IOAC. Here, task performance under FOAC and IOAC is further compared in a more complicated pHRI scenario where contact interactions with a nonlinear, viscoelastic, and layered environment are investigated. Needle insertion experiments were performed with soft silicon samples and task performances under FOAC and IOAC were compared using the metrics defined in Section 4.2.

Needle insertion is commonly used in many medical procedures including biopsies, brachytherapy, injection therapy, and epidural anesthesia. The insertion of a needle into soft tissue involves distinct phases [29]. The process starts with the deformation of the soft tissue under the force exerted by the needle. This deformation continues until rupture occurs, the stage of pure deformation ends, a quick drop in force is observed, and a mixed stage of penetration and deformation starts. At this stage, as the needle continues its movement through the soft tissue, the forces tend to increase until the needle reaches to another layer or comes to a full stop. During the procedure, the operator advances the needle into tissue by appreciating the magnitude of these interaction forces.

In our scenario, the human operator advances the needle into two silicon samples, placed back to back to imitate a layered soft tissue, while the robot keeps the needle straight on a horizontal path (see Fig. 6). Two silicon samples having different stiffnesses were used for the experiments. The samples were prepared using Smooth-Sil 910, which is a two-component silicone rubber: Part A is the base material that forms the product and Part B is the catalyst that hardens the silicon. For the softness adjustment, Silicon Oil was used. To prepare the samples, Parts A, B, and the oil were mixed in two different ratios, and the mixture was poured into cylindrical glass pots for molding. The diameter of the samples S1 and S2 is $45 \mathrm{~mm}$ and their heights are 35 and 20 $\mathrm{mm}$, respectively.

\subsection{Material Characterization of Silicon Samples}

In order to characterize the material properties of the silicon samples, compression experiments were performed using a flat plate attached to the end-effector of the robot (see Fig. 7a). The samples S1 and S2 were compressed to a depth of $10 \mathrm{~mm}$ and $2 \mathrm{~mm}$, respectively, at a rate of $0.05 \mathrm{~mm} / \mathrm{s}$. The compression rate was kept low to reduce the influence of viscoelastic and inertial effects and obtain hyperelastic behavior of the samples. The force response was measured

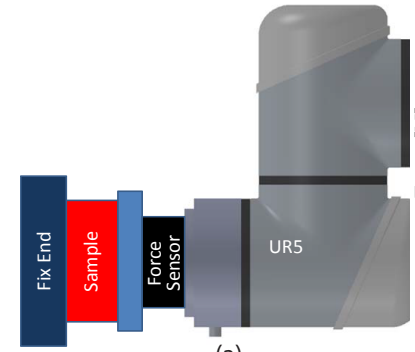

(a)

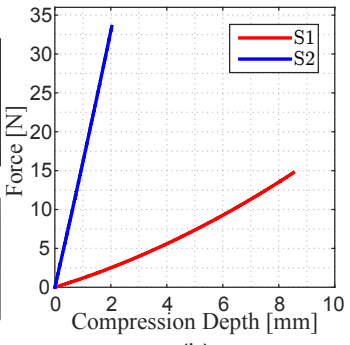

(b)
Fig. 7. (a) Experimental setup for characterization of silicon samples, (b) force vs compression depth curves for two different samples.

by the force sensor on the robot. We fitted a second order polynomial to force response vs compression depth data for better visualisation of the nonlinear elastic behavior of the silicon samples (Fig. 7b). As shown in Fig. 7b, S1 is much softer than S2.

\subsection{Experimental Procedure}

During the experiments, the subject inserted the needle into the samples S1 and S2, placed back to back as shown in Fig. 6, at a rate of $5 \mathrm{~mm} / \mathrm{s}$ (chosen in light of [30]) until a depth of $45 \mathrm{~mm}$ was reached (i.e. ramp phase). Hence, the needle passed through the soft sample (S1) and partially penetrated into the stiffer one (S2). After the desired depth was reached, the hold phase started, and then, the subject maintained the position of the needle at this depth for 10 seconds. A visual feedback showing the needle tip and a cursor moving with a speed of $5 \mathrm{~mm} / \mathrm{s}$ was displayed to the subject through a computer screen, so that the subject inserted the needle at a relatively constant velocity during the ramp phase by following the motion of the visual cursor. The experiment was conducted for 3 different integration orders $\alpha=0.4,0.7$ (FOAC) and 1 (IOAC). The subject repeated the procedure 5 times for each integration order. For consistency with the parameters used in Section 4, admittance controller parameters are set to $m_{F}=20 \mathrm{kgs}^{\alpha-1}$, $m_{I}=20 \mathrm{~kg}$, and $b_{F}=b_{I}=25 \mathrm{Ns} / \mathrm{m}$.

\subsection{Data Collection and Metrics}

For the experiment reported in Section 4, the contact environment was a linear spring having a constant coefficient. Hence, the human force was linearly dependent on the interaction force. However, in needle insertion task, the contact environment (i.e. silicon samples) exhibits nonlinear and layered behaviour. Moreover, as the needle passes through the silicon samples, local stiffness changes as a function of penetration depth. Hence, the human force is not linearly dependent on the interaction force and has to be measured separately. For this reason, we modified the handle design and attached a second force sensor (Mini 40, ATI Inc.) to measure the force applied by human $F_{h}$ directly (Fig. 6). We use the similar metrics defined in Section 4.2 to quantify the human effort under different task conditions. Additionally, we would like to note that $P^{\text {ave }}$ was calculated using directly $F_{h}$ since it was measured by the additional force sensor.

\subsection{Results}

The performance metrics were calculated for the both phases (except $A_{P}$, which was evaluated for the hold phase 
only) of all trials. The means and the standard errors of the means are shown in Fig. 8. End-effector position and velocity, interaction force, and force applied by human during an experimental trial are shown in Fig. 9.
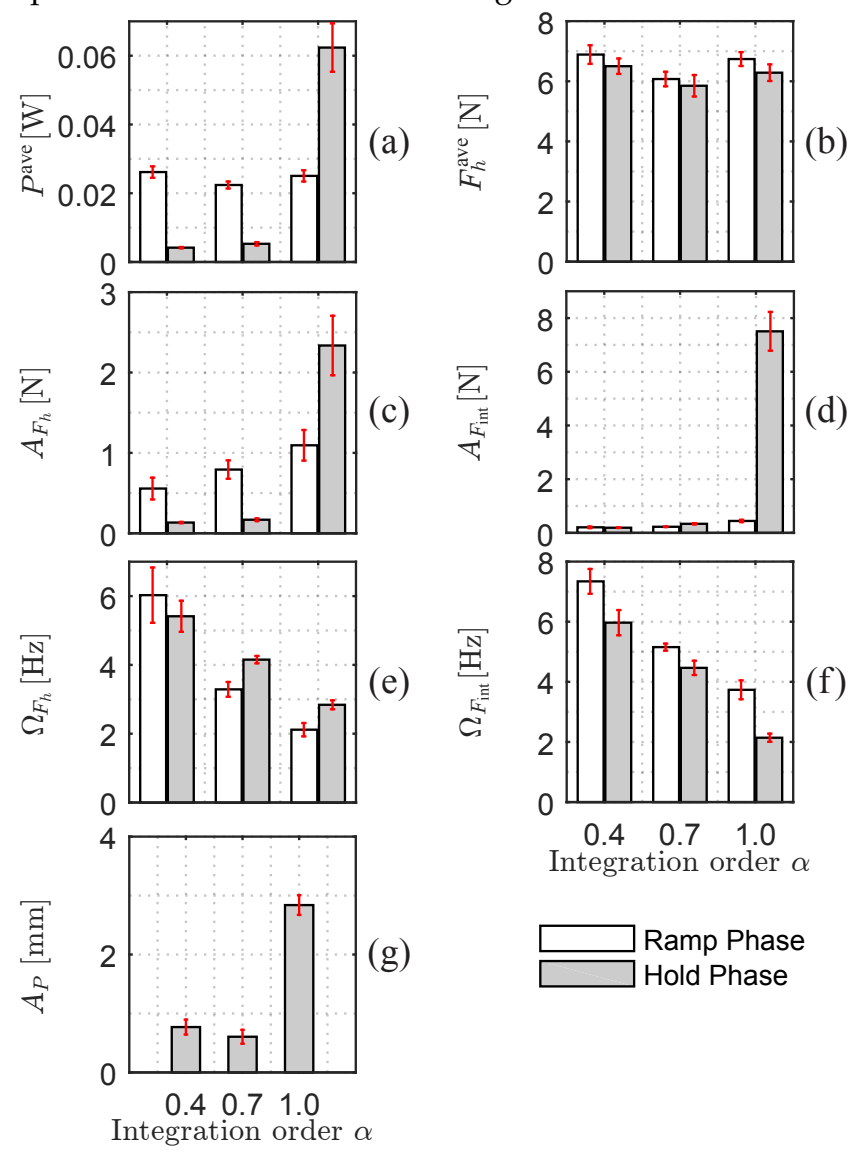

Fig. 8. The means and the standard errors of means of normalized performance metrics for needle insertion experiment.

As shown in Fig. 8a, average power consumed by human, $P^{\text {ave }}$, did not change with the integration order during the ramp phase, whereas it was much higher under IOAC than that of FOAC during the hold phase. Fig. 8b shows that average force $F_{h}^{\text {ave }}$ applied by human was similar for different values of integration order, but the oscillation amplitudes were quite different (Fig. 8c). As shown in Fig. 9d, human force fluctuated significantly during the hold phase of the task under IOAC $(\alpha=1)$. In fact, interaction force under IOAC oscillated even with a higher amplitude during the hold phase (Figs. 8d and 9c). Average dominant frequency of oscillations in force applied by human, $\Omega_{F_{h}}$, and interaction force, $\Omega_{F_{\text {int }}}$, increased as the integration order $\alpha$ decreased during both phases (Figs. 8e and 8f). Average amplitude of oscillations in end-effector position $A_{P}$ was higher under IOAC during the hold phase (Fig. 8g). On the other hand, the change in the integration order did not affect $A_{P}$ much under FOAC. We can observe that the amplitude of oscillations in interaction force, force applied by human, and end-effector position and velocity were larger under IOAC than that of FOAC during the hold phase (Fig. 9).

\subsection{Discussion}

During needle insertion, achieving higher target precision is required to avoid tissue damage [30]. Large oscillation amplitudes in force and position can cause larger deviations

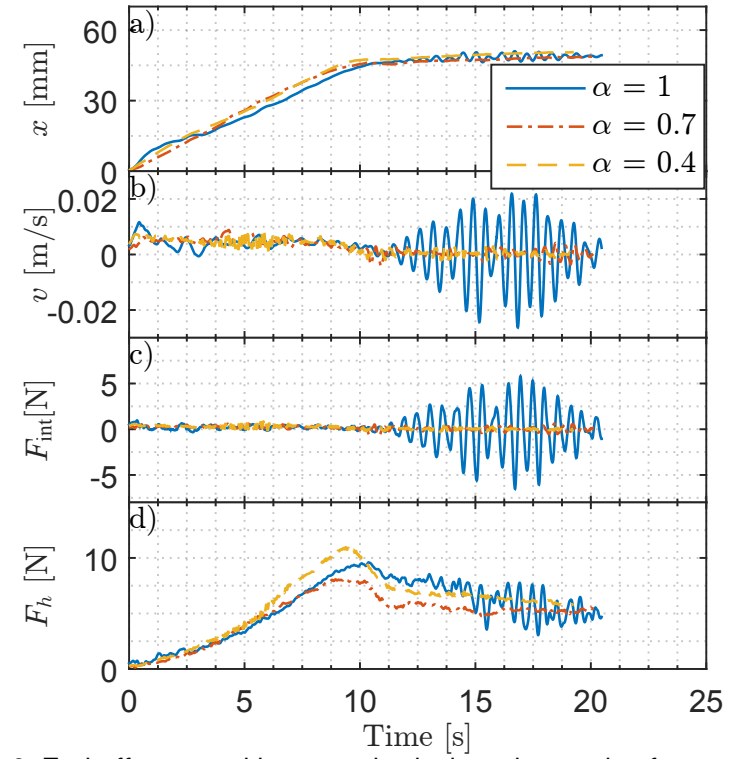

Fig. 9. End-effector position $x$, and velocity $v$, interaction force $F_{\text {int }}$, force applied by human $F_{h}$ as a function of time.

of needle tip from the target region. Furthermore, if needle is to be inserted into critical and sensitive regions in human body, undesirable motion of the needle should be avoided to reduce harming neighboring tissue. For instance, the epidural space in an adult lumber spine is only 3-5 $\mathrm{mm}$ thick, and unintentional dural puncture, causing complications, may occur during epidural injection while operator attempts to identify the epidural space [31], [32]. Our experiment shows that, compared to IOAC, FOAC is better in task precision since the fluctuations in needle tip were dampened (see Fig. 9) more effectively during the hold phase. The position, velocity, and force profiles shown in Fig. 9 were consistent in all insertion trials.

Similar to the experimental results reported in Section 4.4, frequency of oscillations in force slightly increased under FOAC (Figs. 8e and 8f), but damping remained effective for larger range of frequencies (as anticipated from Fig. 3c), and hence, needle tip oscillations were better absorbed (Fig. 8g). Using IOAC with higher admittance damping would also reduce oscillations, but higher human effort would be required to insert the needle during the ramp phase under high admittance damping. On the other hand, FOAC displays frequency-dependent damping, which enforces higher effective damping for the high frequency components of interaction force (see Fig. 3c).

The results of the needle insertion experiments show that human effort was reduced under FOAC. The power consumed by human did not change during the ramp phase for different values of integration order, while it was significantly reduced during the hold phase (Fig. 8a). In general, reduction in amplitude of oscillations in force and end-effector position, as well as power consumed by human under FOAC during the hold phase is inline with the results of the first experiment reported in Section 4. Augmenting our control architecture given in Fig. 1 by taking advantage of additional techniques such as force amplification [33] may also be beneficial for further reducing the human effort. However, in our experiments, we did not utilize such additional methods since our aim here is to show clearly that 
FOAC reduces the human effort and outperforms IOAC.

An experimental comparison of FOAC and IOAC is challenging since parameters of FOAC are not physically equivalent to those of IOAC. The controller parameters of FOAC can be mapped to parameters of IOAC through impedance matching at a particular frequency such that responses of two controllers can be compared. During the ramp phase of our experiments, subjects were asked to move the end-effector at a constant velocity, so the desired operating frequency of the motion is zero. Given that task frequency is zero, we performed impedance matching at this frequency, such that FOAC and IOAC parameters are set to be equal at this frequency, resulting in $m_{F}=m_{I}$ and $b_{F}=b_{I}$. Note that the value of admittance mass does not affect the response at zero frequency. Consequently, as the integration order changes, $A_{F_{h}}$, during the ramp phase, should not change ideally, but during the experiments changes are observed since users cannot maintain a perfectly constant speed. Moreover, $A_{F_{h}}$ and $A_{P}$ under these controllers differ more during the hold phase since FOAC dampens these oscillations more effectively.

\section{Conclusion}

In this study, we showed potential advantages of FOAC over IOAC. Given the admittance controller affects the coupled system dynamics in a non-trivial manner, rendering analytical design methods infeasible, a computational approach is used to determine appropriate controller parameters for bounded human and environment impedances. Our computational approach resembles to the complementary stability analysis in [19]. The stability analysis, carried out for a bounded equivalent impedance, revealed that the stability robustness of control parameters to changes in human and/or environment stiffness was improved under FOAC.

In addition to the stability maps, we studied transparency, stability robustness, and task performance through two pHRI studies involving continuous contact with linear and nonlinear environments. Under impedance matching, we analytically showed that the stability robustness under FOAC is higher than that of IOAC for any environment and human arm impedance. We also showed that FOAC provides advantages in terms of the transparency of the closed loop system. In particular, the computational analysis of the closed loop impedance of the coupled system revealed that the effective damping of the closed loop system under IOAC decays as the frequency increases. However, effective damping under FOAC increases up to a certain frequency, before it starts to decay. In other words, damping supplied to the coupled system under FOAC remains large for an extended range of frequencies. We showed that this frequencydependent damping behavior of FOAC is beneficial for ensuring robust stability. Furthermore, our experimental results provided evidence that this behavior under FOAC enhanced human performance by absorbing vibrations during pHRI tasks involving physical interactions with linear and nonlinear environments.

Impedance matching analysis revealed that the parameters for FOAC maps to different parameters of IOAC at each frequency. The frequency dependent nature of FOAC is advantageous as it enhances the stability robustness of the system at high frequency ranges, while providing favorable transparency characteristics at low frequency ranges. Note that similar, and even more favorable, system responses can be achieved through the use of adaptive IOAC. However, as the controller parameters of such an adaptive controller would be frequency dependent, an adaptive IOAC will not be LTI. As a result, methods/tools from the familiar linear systems theory would not be applicable for designing such controllers, significantly complicating their analysis. Hence, one of the main advantages of FOAC is the fact that it can provide frequency dependent controller parameters while its design/analysis can still be handled within the relatively simple LTI systems framework.

Although FOAC showed robustness to changes in human arm and environment stiffness, time-variant behavior of human arm is neglected in all our analyses. Similarly, adaptive controllers are not considered in this work, such that LTI systems analysis can be used throughout the paper. Time varying nature of human/environment and adaptive admittance controller are planned to be the focus of our future studies. In particular, adaptive approaches will be investigated for FOAC to alter the admittance controller parameters on-the-fly based on the changes in human intention during pHRI tasks. Human intention can be estimated using machine learning as in [34] to adjust the controller parameters adaptively. Moreover, integration of our earlier work on i) load sharing [1], and ii) role exchange [35] mechanisms into an adaptive FOAC may lead to richer interactions for $\mathrm{pHRI}$.

\section{ACKNOWLEDGMENTS}

The authors would like to thank to Prof. A. Kucukyilmaz for her valuable comments. Moreover, Y.A. thanks A. Aytekin, E.S. Emgin, and O. Caldiran for fruitful discussions during the study.

\section{REFERENCES}

[1] A. Mörtl, M. Lawitzky, A. Kucukyilmaz, M. Sezgin, C. Basdogan, and S. Hirche, "The role of roles: Physical cooperation between humans and robots," The International Journal of Robotics Research, vol. 31, no. 13, pp. 1656-1674, 2012.

[2] T. Wojtara, M. Uchihara, H. Murayama, S. Shimoda, S. Sakai, H. Fujimoto, and H. Kimura, "Human-robot collaboration in precise positioning of a three-dimensional object," Automatica, vol. 45, no. 2, pp. $333-342,2009$.

[3] N. Hogan, "Impedance control: An approach to manipulation," in American Control Conference, 1984, pp. 304-313.

[4] F. Dimeas and N. Aspragathos, "Online stability in human-robot cooperation with admittance control," IEEE Transactions on Haptics, vol. 9, no. 2, pp. 267-278, 2016.

[5] T. Tsumugiwa, R. Yokogawa, and K. Yoshida, "Stability analysis for impedance control of robot for human-robot cooperative task system," in IEEE/RSJ International Conference on Intelligent Robots and Systems, vol. 4, 2004, pp. 3883-3888.

[6] V. Duchaine and C. M. Gosselin, "Investigation of human-robot interaction stability using lyapunov theory," in IEEE International Conference on Robotics and Automation, 2008, pp. 2189-2194.

[7] W. Gallagher, D. Gao, and J. Ueda, "Improved stability of haptic humanrobot interfaces using measurement of human arm stiffness," Advanced Robotics, vol. 28, no. 13, pp. 869-882, 2014.

[8] X. Lamy, F. Colledani, F. Geffard, Y. Measson, and G. Morel, "Achieving efficient and stable comanipulation through adaptation to changes in human arm impedance," in IEEE International Conference on Robotics and Automation, May 2009, pp. 265-271.

[9] J. E. Colgate and N. Hogan, "Robust control of dynamically interacting systems," International Journal of Control, vol. 48, no. 1, pp. 65-88, 1988. 
[10] J. E. Colgate and G. G. Schenkel, "Passivity of a class of sampleddata systems: Application to haptic interfaces," Journal of Robotic Systems, vol. 14, no. 1, pp. 37-47, 1997.

[11] T. Hulin, A. Albu-Schffer, and G. Hirzinger, "Passivity and stability boundaries for haptic systems with time delay," IEEE Transactions on Control Systems Technology, vol. 22, no. 4, pp. 1297-1309, July 2014.

[12] B. Hannaford and J.-H. Ryu, "Time-domain passivity control of haptic interfaces," IEEE Transactions on Robotics and Automation, vol. 18, no. 1, pp. 1-10, Feb 2002.

[13] J.-H. Ryu, D.-S. Kwon, and B. Hannaford, "Stable teleoperation with time-domain passivity control," IEEE Transactions on Robotics and Automation, vol. 20, no. 2, pp. 365-373, April 2004.

[14] - "Stability guaranteed control: time domain passivity approach," IEEE Transactions on Control Systems Technology, vol. 12, no. 6, pp. 860-868, Nov 2004.

[15] T. Tsuji, P. G. Morasso, K. Goto, and K. Ito, "Human hand impedance characteristics during maintained posture," Biological Cybernetics, vol. 72, no. 6, pp. 475-485, 1995.

[16] J. M. Dolan, M. B. Friedman, and M. L. Nagurka, "Dynamic and loaded impedance components in the maintenance of human arm posture," IEEE Transactions on Systems, Man, and Cybernetics, vol. 23, no. 3, pp. 698-709, May 1993.

[17] A. Haddadi and K. Hashtrudi-Zaad, "Bounded-impedance absolute stability of bilateral teleoperation control systems," IEEE Transactions on Haptics, vol. 3, no. 1, pp. 15-27, 2010.

[18] S. P. Buerger, H. I. Krebs, and N. Hogan, "Characterization and control of a screw-driven robot for neurorehabilitation," in IEEE International Conference on Control Applications, 2001, pp. 388-394.

[19] S. P. Buerger and N. Hogan, "Complementary stability and loop shaping for improved human-robot interaction," IEEE Transactions on Robotics, vol. 23, no. 2, pp. 232-244, April 2007.

[20] Y. Aydin, O. Tokatli, V. Patoglu, and C. Basdogan, "Fractional order admittance control for physical human-robot interaction," in IEEE World Haptics Conference (WHC), June 2017, pp. 257-262.

[21] M. Ayyildiz, C. Basdogan, and R. G. Aktas, "Effect of solution and post-mortem time on mechanical and histological properties of liver during cold preservation." Biorheology, vol. 51, no. 1, pp. 47-70, 2014.

[22] O. Tokatli and V. Patoglu, "Stability of haptic systems with fractional order controllers," in IEEE/RSJ International Conference on Intelligent Robots and Systems, Sept 2015, pp. 1172-1177.

[23] - "Using fractional order elements for haptic rendering," in ISRR International Symposium on Robotics Research, 2015.

[24] Y. Chen, I. Petras, and D. Xue, "Fractional order control - a tutorial," in American Control Conference, 2009, pp. 1397-1411.

[25] J. S. Mehling, J. E. Colgate, and M. A. Peshkin, "Increasing the impedance range of a haptic display by adding electrical damping," in First Joint Eurohaptics Conference and Symposium on Haptic Interfaces for Virtual Environment and Teleoperator Systems, 2005, pp. 257-262.

[26] N. Colonnese, A. F. Siu, C. M. Abbott, and A. M. Okamura, "Rendered and characterized closed-loop accuracy of impedancetype haptic displays," IEEE Transactions on Haptics, vol. 8, no. 4, pp. 434-446, Oct 2015.

[27] D. Matignon, "Generalized fractional differential and difference equations: stability properties and modelling issues," in Mathematical Theory of Networks and Systems symposium, 1998, pp. 503-506.

[28] K. J. Åström and R. M. Murray, Feedback Systems: An Introduction for Scientists and Engineers. Princeton university press, 2010.

[29] C. Gokgol, C. Basdogan, and D. Canadinc, "Estimation of fracture toughness of liver tissue: Experiments and validation," Medical Engineering $\mathcal{E}$ Physics, vol. 34, no. 7, pp. 882 - 891, 2012.

[30] T. K. Podder, D. P. Clark, D. Fuller, J. Sherman, W. S. Ng, L. Liao, D. J. Rubens, J. G. Strang, E. M. Messing, Y. D. Zhang, and Y. Yu, "Effects of velocity modulation during surgical needle insertion," in IEEE Engineering in Medicine and Biology 27th Annual Conference, 2005, pp. 5766-5770.

[31] J. S. Sprigge and S. J. Harper, "Accidental dural puncture and post dural puncture headache in obstetric anaesthesia: presentation and management: A 23-year survey in a district general hospital," Anaesthesia, vol. 63, no. 1, pp. 36-43, 2008.

[32] M. C. Norris, B. L. Leighton, and C. A. DeSimone, "Needle bevel direction and headache after inadvertent dural puncture," Anesthesiology, vol. 70, no. 5, pp. 729-731, 1989.

[33] X. Lamy, F. Colldani, F. Geffard, Y. Measson, and G. Morel, “Human force amplification with industrial robot : Study of dynamic limitations," in IEEE/RSJ International Conference on Intelligent Robots and Systems, Oct 2010, pp. 2487-2494.

[34] C. E. Madan, A. Kucukyilmaz, T. M. Sezgin, and C. Basdogan, "Recognition of haptic interaction patterns in dyadic joint object manipulation," IEEE Transactions on Haptics, vol. 8, no. 1, pp. 5466, Jan 2015.

[35] A. Kucukyilmaz, T. M. Sezgin, and C. Basdogan, "Intention recognition for dynamic role exchange in haptic collaboration," IEEE Transactions on Haptics, vol. 6, no. 1, pp. 58-68, 2013.

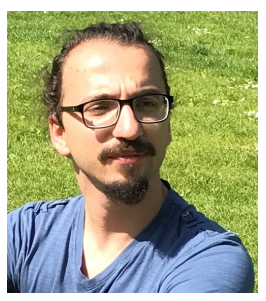

Yusuf Aydin Yusuf Aydin is currently a PhD candidate in mechanical engineering department at Koc University, Istanbul. He received his BSc dual degree in mechanical engineering and electrical and electronics engineering, and then, MSc degree in mechanical engineering from Koc University, Istanbul, in 2011, and 2013 respectively. His research interests include physical human-robot interaction, haptics, robotics, control, and mechatronics.

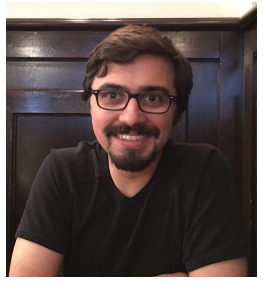

Ozan Tokatli Ozan Tokatli recieved his BSc, MSc and PhD degrees in Mechatronics Engineering from Sabanci University in 2008, 2010 and 2015, respectively. He is currently a post doctoral research associate in Haptics Laboratory at University of Reading. During his PhD, he worked on haptic systems with fractional order controllers. His current research interest is utilizing haptics technology for enhanced learning in schools. His research interests extend to design tics and pHRI. of haptic interfaces and control of robots for hap-

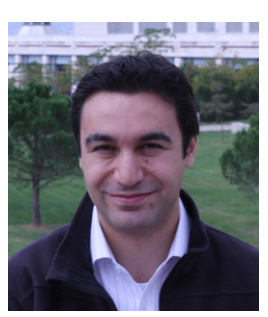

Volkan Patoglu Volkan Patoglu received his Ph.D. degree in Mechanical Engineering from the University of Michigan, Ann Arbor in 2005. He worked as a post doctoral research associate at Rice University. Currently, he is an Associate Professor at Sabanci University. His research is in the area of physical human-machine interaction, in particular, design and control of force feedback robotic systems with applications to rehabilitation and skill training. His research extends to cognitive robotics. Dr. Patoglu serves as an Associate Editor for the IEEE Transactions on Haptics.

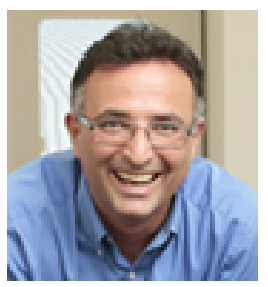

Cagatay Basdogan Cagatay Basdogan received the PhD degree in mechanical engineering from Southern Methodist University in 1994. $\mathrm{He}$ is a faculty member in the mechanical engineering and computational sciences and engineering programs of Koc University, Istanbul, Turkey. He is also the director of the Robotics and Mechatronics Laboratory at Koc University. Before joining Koc University, he worked at NASA-JPL/Caltech, MIT, and Northwestern University Research Park. His research interests include haptic interfaces, robotics, mechatronics, biomechanics, medical simulation, computer graphics, and multi-modal virtual environments. $\mathrm{He}$ is currently the associate editor in chief of IEEE Transactions on Haptics and serves in the editorial boards of IEEE Transactions on Mechatronics, Presence: Teleoperators and Virtual Environments, and Computer Animation and Virtual Worlds journals. 